\title{
Criminologie
}

\section{Violence sectaire : un coup de sonde en terre d'Islam}

\section{Sami Aoun}

Volume 41, numéro 2, automne-hiver 2008

Les organisations dites sectes, les lois et la société

URI : https://id.erudit.org/iderudit/019434ar

DOI : https://doi.org/10.7202/019434ar

Aller au sommaire du numéro

Éditeur(s)

Les Presses de l'Université de Montréal

\section{ISSN}

0316-0041 (imprimé)

1492-1367 (numérique)

Découvrir la revue

\section{Citer cet article}

Aoun, S. (2008). Violence sectaire : un coup de sonde en terre d'Islam. Criminologie, 41(2), 91-115. https://doi.org/10.7202/019434ar

\section{Résumé de l'article}

Les actes de violence dans quelques coins du monde musulman révèlent des failles graves dans le contrat social. L'éclosion de la violence sectaire met en cause la solidité de la démarche de la modernisation étatique. La culture arabo-musulmane accuse des symptômes qui la rendent couveuse de violence sectaire : cette dernière est perçue comme une crise de la culture arabo-musulmane, depuis sa formation. Une vision dualiste du monde et exclusiviste de l'Autre, dans laquelle les protomoteurs de la violence sectaire recourent à des argumentaires exclusivistes et appellent à la diabolisation de l'Autre. En plus d'une tendance autodestructrice enveloppée d'une idéologie du martyre, où l'éloge de la vie a été éclipsé par l'éloge de la mort. Sans oublier aussi le constat de l'échec, ou même la faillite du modèle étatique dans cette région. Ajoutons aussi l'intervention étrangère, notamment celle étatsunienne dans le cas de l'Irak, qui peut expliquer partiellement l'instauration d'une telle violence.

Plusieurs autres constats sur le phénomène de la violence sectaire dans l'espace islamique peuvent être soulignés. La citoyenneté, notion centrale de la modernité, n’a pas réussi à prendre sa place comme lien qui prime sur les appartenances religieuses et confessionnelles. Les loyautés primaires (religieuses, communautaires, tribales ou claniques) ont ainsi prévalu sur la loyauté à l'État. Ce qui est noté dans la disparition ou le rétrécissement de l'écart entre l'État et la société civile. Dans ce contexte, la montée des associations religieuses et des groupes tribalo-claniques devient une réalité préoccupante pour l'État. Ces groupes deviennent des refuges et des instances d'autodéfense contre la violence étatique et constituent des alternatives même à l'échec de l'État dans sa fonction de veiller au bien-être de ses citoyens. Le point fondamental pour sortir de ce labyrinthe sectaire : le retour d'un État civil fonctionnel, soit un État de droit doté de mécanismes de résolution de conflits et détenant le monopole exclusif de la violence.
Ce document est protégé par la loi sur le droit d'auteur. L’utilisation des services d’Érudit (y compris la reproduction) est assujettie à sa politique d'utilisation que vous pouvez consulter en ligne.

https://apropos.erudit.org/fr/usagers/politique-dutilisation/ 


\title{
Violence sectaire: un coup de sonde en terre d'Islam
}

\author{
Sami Aoun \\ Professeur \\ École de politique appliquée, Université de Sherbrooke \\ Sami.Aoun@USherbrooke.ca
}

RÉSUMÉ - Les actes de violence dans quelques coins du monde musulman révèlent des failles graves dans le contrat social. L'éclosion de la violence sectaire met en cause la solidité de la démarche de la modernisation étatique. La culture arabo-musulmane accuse des symptômes qui la rendent couveuse de violence sectaire: cette dernière est perçue comme une crise de la culture arabo-musulmane, depuis sa formation. Une vision dualiste du monde et exclusiviste de l'Autre, dans laquelle les protomoteurs de la violence sectaire recourent à des argumentaires exclusivistes et appellent à la diabolisation de l'Autre. En plus d'une tendance autodestructrice enveloppée d'une idéologie du martyre, où l'éloge de la vie a été éclipsé par l'éloge de la mort. Sans oublier aussi le constat de l'échec, ou même la faillite du modèle étatique dans cette région. Ajoutons aussi l'intervention étrangère, notamment celle étatsunienne dans le cas de l'Irak, qui peut expliquer partiellement l'instauration d'une telle violence.

Plusieurs autres constats sur le phénomène de la violence sectaire dans l'espace islamique peuvent être soulignés. La citoyenneté, notion centrale de la modernité, n'a pas réussi à prendre sa place comme lien qui prime sur les appartenances religieuses et confessionnelles. Les loyautés primaires (religieuses, communautaires, tribales ou claniques) ont ainsi prévalu sur la loyauté à l'État. Ce qui est noté dans la disparition ou le rétrécissement de l'écart entre l'État et la société civile. Dans ce contexte, la montée des associations religieuses et des groupes tribalo-claniques devient une réalité préoccupante pour l'État. Ces groupes deviennent des refuges et des instances d'autodéfense contre la violence étatique et constituent des alternatives même à l'échec de l'État dans sa fonction de veiller au bien-être de ses citoyens. Le point fondamental pour sortir de ce labyrinthe sectaire: le retour d'un État civil fonctionnel, soit un État de droit doté de mécanismes de résolution de conflits et détenant le monopole exclusif de la violence. 


\section{Introduction}

Les actes de violence sectaire au sein des foyers de troubles ou au cours des guerres intra-étatiques dans quelques coins du monde musulman ${ }^{1}$ interpellent un regard critique sur ce type de violence. Il est impératif de circonscrire les facteurs déclencheurs d'une violence, à la fois religieuse, confessionnelle et communautaire qui fait effondrer l'ordre étatique et apte à le désarticuler ou même à le rendre impossible.

Ces actes de violence révèlent des failles graves dans le contrat social et des blocages dans la citoyenneté et surtout dans la formation de l'État de droit dans les entités musulmanes. L'éclosion de la violence sectaire met en cause la solidité de la démarche de la modernisation étatique et révèle la nature de la résistance aux influences hégémoniques des puissances occidentales.

Pour bien comprendre le pourquoi de la violence sectaire au nom de l'Islam et dans les pays musulmans ou à majorité musulmane, le

1. Maxime Rodinson stipule dans son ouvrage Marxisme et monde musulman:

«On peut entendre par "monde musulman", l'ensemble cohérent de pays et de régions où les musulmans forment au moins une partie importante de la population. On peut distinguer, à l'intérieur de cette entité, un «bloc d'États musulmans» c'est-à-dire d'États indépendants à majorité musulmane, ce bloc forme en réalité deux groupes cohérents inégaux, le groupe malayo-indonésien séparé du groupe que forme tout le reste par une vaste région (Inde, Birmanie, Thaïlande, etc.) où l'islam est en minorité. Ce dernier groupe enserre deux îlots: Israël à majorité non musulmane et le Liban à moitié chrétien ou à faible majorité non musulmane, mais où l'égalité fictive des confessions est postulée (plus Ceuta et Melilla chrétiens et espagnols). Les parties du monde musulman en dehors du bloc des États musulmans peuvent être tenues pour une frange extérieure de celui-ci.» (Rodinson, 1972: 26)

Cette définition donnée par M. Rodinson en 1972 reste plus ou moins valable pour le monde musulman aujourd'hui, même si on trouve chez d'autres auteurs une définition plus large dépassant le cadre géographique. En effet, le juriste marocain Abdelhadi Boutaleb stipule:

«Avant toute considération géographique, économique, sociale, nous pouvons définir le monde islamique comme étant celui de la mission universelle de Mohammed. Son message s'adresse à l'humanité toute entière [...]. La communauté musulmane en charge de ce message est constituée des groupes de musulmans établis à travers le monde et unis par la révélation mohammadienne.» (Boutalbeb, 1995: 55)

Donc, à toutes fins utiles, on peut dire que le monde musulman actuel est formé par tous les pays adhérents à l'Organisation de la conférence islamique (OCI). Cette dernière compte 57 États. Ces États sont: Afghanistan, Albanie, Algérie, Arabie saoudite, Azerbaïdjan, Bahrain, Bangladesh, Bénin, Brunei, Burkina-Faso, Cameroun, Comores, Côte d'Ivoire, Djibouti, Égypte, Émirats arabes unis, Gabon, Gambie, Guinée, Guinée-Bissau, Guyane, Indonésie, Iran, Iraq, Jordanie, Kazakhstan, Kirghizstan, Liban, Libye, Malaisie, Maldives, Mali, Mauritanie, Maroc, Mozambique, Niger, Nigeria, Koweit, Oman, Pakistan, Palestine, Qatar, Sénégal, Sierra Leone, Somalie, Soudan, Suriname, Syrie, Tadjikistan, Tchad, Togo, Tunisie, Turquie, Turkménistan, Ouganda, Ouzbékistan, Yémen. 
concours des facteurs exogènes et endogènes qui couvent et entretiennent cette forme d'horreur constitue le pilier de l'analyse que nous proposons. Ainsi, cette réflexion se veut une contribution à la compréhension du phénomène de la violence sectaire dans l'espace arabomusulman. Le cas d'analyse mis en étude sera celui connu en Irak après la chute du président Saddam Hussein en 2003. Dans ce pays, la violence sectaire constitue une réalité au quotidien. Une violence sectaire qui s'inscrit, en outre, dans le contexte de la présence militaire étrangère en Irak au lendemain de la fin de la dictature de Saddam Hussein. À vrai dire, la violence sectaire chevauche les domaines de la guerre de libération, de la guerre civile et de la guerre religieuse et ethnique. Le cas de l'Irak, qui sera pertinent pour cette réflexion, est surtout celui de la polarisation sunnite/chiite sans toutefois ignorer les cas de violence contre les minorités ethno-religieuses chrétiennes, kurdes, yézidis ou turcomanes.

Le présent article présentera d'abord un survol géopolitique du monde musulman en conflit, puis examinera les facteurs exogènes qui soutiennent la violence sectaire, ensuite discutera des facteurs endogènes et des discours et méthodes de la violence sectaire en Irak avant de conclure. Mais avant, il est utile de remarquer que la culture arabo-musulmane accuse des symptômes de facteurs qui la rendent couveuse de violence sectaire. Entre autres symptômes, les suivants seront développés:

- Un Patrimoine mis en cause: où la violence sectaire est perçue comme une crise de la culture arabo-musulmane, depuis sa formation.

- Une vision dualiste du monde et exclusiviste de l'Autre: les intégristes de la violence sectaire recourent à des argumentaires exclusivistes et appellent à la diabolisation de l'Autre. Les intégristes violents sunnites perçoivent ainsi les chiites comme des Rawafid (négationnistes du vrai appel de l'islam). Alors que les intégristes chiites accusent les sunnites d'être des Nawasib, soit des ennemis d'Al al-bayt ce qui signifie «les Gens ou la Famille de la maison». Selon la tradition islamique, cette expression se rapporte plus précisément à la famille du Prophète et à ses descendants, et du coup signifie des ennemis de Dieu. Dans les deux cas, la cohabitation entre les deux branches de l'islam devient difficile, et le recours à la violence sectaire devient probable.

- Une tendance autodestructrice enveloppée d'une idéologie du martyre. L'éloge de la vie a été éclipsé par l'éloge de la mort et du sacrifice en vue de créer l'unité de l'Umma. Cette idéologie du martyre «s'allie à un mépris du corps, de la douleur, de la vie matérielle et de l'univers social» (Mayeur-Jaouen, 2002). Dans cette perspective, la violence sectaire est 
un dérapage de l'incapacité de répondre aux défis de la domination extérieure par les moyens conventionnels, les exigences de la guerre asymétrique ayant en effet favorisé le recours aux attentats suicides. Paradoxalement le même procédé a été fréquemment utilisé contre les autres musulmans! Ce qui l'a rendu un instrument de violence sectaire.

Il ne faut pas perdre de vue que la violence est un des éléments constitutifs de l'histoire musulmane, sous forme de pénalités, de jibad ${ }^{2} .$. etc. Sauf qu'il s'agit alors d'une violence codifiée, qui n'est pas exclusive aux pays majoritairement musulmans. Mais l'usage de la violence est ambigu faute soit de légitimité de l'acteur, soit de l'abus de l'argumentaire. Cela étant, et avant d'essayer d'analyser ces trois formes appartenant aux dimensions socioculturelle et psychologique, il est adéquat de mettre l'accent sur le concours de deux dynamiques qui ont rendu possible l'éclosion de la violence sectaire dans l'espace musulman. Ces deux dynamiques sont constituées par:

- L'échec, ou même la faillite du modèle étatique dans cette région soit à se débarrasser de son lien avec le sectarisme, soit à créer des mécanismes pour régler les débordements de la violence qui, en raison de son abri sous la coupole de la dictature et l'oppression, verrouille l'espace économique et social.

- L'intervention étrangère, notamment étatsunienne dans le cas de l'Irak, peut expliquer partiellement l'instauration d'une telle violence. En effet, il est évident que cette invasion se déroule dans un contexte géopolitique explosif (intervention militaire des puissances étrangère, etc.) et dans un environnement culturel de confrontation (instabilité constructive $^{3}$, choc des religions, etc.). Du même souffle, faut-il mentionner les intrusions régionales, en particulier la polarisation entre les deux puissances musulmanes: l'Iran chiite et l'Arabie saoudite sunnite dans leur quête du leadership régional actuel. Cette quête par les États arabes dont la Syrie, l'Égypte, la Libye et l'Arabie saoudite a d'ailleurs déjà été une source d'instabilité dans la région à partir des années 1950. Elle est devenue plus palpable toutefois depuis la disparition des chefs charismatiques (à l'instar d'un Nasser, par exemple), ce qui a abouti à la menace d'une balkanisation de l'espace arabo-musulman.

2. Il est important de ne pas confondre la violence dite légitime, selon les termes de Max Weber, exercée par l'État selon ses lois, et celle utilisée par des individus/groupes contre l'État ou d'autres individus/groupes.

3. Qui dépeint «le monde arabe comme un agrégat de minorités religieuses et ethniques incapables de vivre ensemble dans des entités étatico-nationales», selon les termes de Walid Charara (2005). 
Néanmoins, le concours de tels facteurs exogènes, qui seront discutés plus avant, reste plus ou moins mineur dans l'enclenchement de la violence sectaire dans cet espace qui est déjà fragilisé par ses guerres intestines éclatées ou latentes ainsi que par des facteurs endogènes qui s'imposent comme des causes majeures de cette violence et que nous aborderons plus loin.

\section{Survol géopolitique du monde musulman en termes de conflits}

Dans cette perspective, deux courants s'opposent. Une première approche met toujours la faute sur les étrangers, ou selon l'expression de l'homme politique et journaliste libanais Ghassan Tueni, au sujet de la guerre civile libanaise: "c'est la guerre des autres et non la nôtre» (Tueni, 1985). La seconde approche met l'accent sur les problèmes internes de l'espace arabo-musulman, c'est-à-dire ceux qui l'affaiblissent et le rendent «colonisable» selon les termes du penseur algérien Malek Bennabi (1990).

Depuis la fin de la période colonialiste, au début des années 1960, l'espace arabo-musulman connaît en effet des conflits internes, avec de fortes tensions idéologiques surtout autour des questions d'identité. Plusieurs tensions constituent des foyers de crispation mobilisant des symboles identitaires et religieux, drainant les ressources et laissant des effets négatifs sur les projets de développement économique et social dans les pays du Moyen-Orient. Cela est d'autant plus le cas que l'espace arabo-musulman, qui regorge de ressources énergétiques, est non seulement devenu une priorité pour la politique étrangère étatsunienne, mais est aussi un champ de rivalités entre les grandes puissances pour des raisons économiques et idéologiques. Cette région a d'ailleurs connu, dès sa formation et tout au long de la guerre froide, des conflits marquants comme les guerres israélo-arabes et les guerres du Golfe. Il existe aussi d'autres tensions entre les pays qui s'avèrent plus difficiles à résoudre. À la suite de la guerre contre le terrorisme au lendemain du 11 septembre 2001, l'espace arabo-musulman serait en outre sujet à de nouvelles pressions, jusque-là inédites. Il faut noter que les crises internes des États telles que les limitations de légitimité, la crispation entre les composantes ethniques, ou encore entre majorités et minorités (sunnites, chiites, chrétiens) se dressent comme un obstacle sérieux à la résolution des conflits. 
Ces conflits sont devenus une occasion de mobilisation des symboles identitaires et religieux. Ces tensions drainent plusieurs ressources et affectent négativement les projets de développement économique et social. D'ailleurs, l'absence ou les dérapages du développement humanitaire seraient largement responsables de la violence sectaire. On imagine mal un conflit à base sectaire dans un pays avec un indice de développement humain meilleur. Les situations actuelles illustrent bien ce déficit :

- Le cas de la guerre du Liban (1975-1990) est l'exemple type d'un positionnement symbolique et identitaire qui s'appuie sur des dimensions confessionnelles et religieuses, même si les causes profondes pourraient être, entre autres, politiques, sécuritaires, sociales et économiques.

- L'Irak après Saddam Hussein est un autre exemple où l'absence d'un chef charismatique et l'effondrement du système totalitaire déclenche une violence sectaire déjà latente, dans une société maîtrisée par le fer et le sang.

- Au Cachemire, où le conflit absorbe une grande partie des potentialités du Pakistan et de l'Inde, la guerre des mosquées et des rues entre sunnites et chiites reste ininterrompue. Seule la dimension pakistanaise est ici soulignée cependant, car l'Inde n'appartient pas au monde arabomusulman.

- Banda Aceh: le conflit armé, opposant le gouvernement de Jakarta en Indonésie aux militants séparatistes du Mouvement Aceh libre (GAM), depuis 1976, risque de prendre des proportions plus grandes, surtout après la séparation du Timor oriental. Toujours est-il que la violence sectaire y est le vecteur identitaire des violences.

- Mindanao: deux groupes revendicatifs cohabitent sur cette île des Philippines, un autonomiste (MNLF), islamiste modéré, et un indépendantiste islamique plus radical (MAILF apparu en 1984). En 1991, la dissidence du groupe Abou Sayaf, renforcé par des combattants arabes, sera responsable des actes les plus violents.

- Le Darfour: la guerre ethnique entre africains et arabes, qui ensanglante le nord-ouest du Soudan depuis 2003 a provoqué une catastrophe humanitaire de grande envergure. En effet, les destructions de villages et une politique de la terre brûlée forcent les populations à la déportation.

D'autres sortes de conflits latents peuvent aussi facilement verser dans la confrontation; ils revêtent tous un caractère idéologique et absorbent les protagonistes dans d'étroits calculs politiques et idéologiques. Parmi eux, on peut citer les rivalités entre l'Iran et l'Arabie saoudite. En fait, 
il s'agit de rivalités pour assurer un leadership régional, bien qu'elles recèlent, au fond, un conflit historique et doctrinal quant aux interprétations coraniques dominantes, qu'il s'agisse par exemple du sunnisme ou du chiisme, qui est minoritaire dans l'espace musulman, mais majoritaire notamment en Iran, au Bahreïn et en Irak.

Les cas actuels de violence sectaire dans l'espace arabo-musulman ont profondément affecté la pensée arabe contemporaine. Ainsi, la guerre du Liban (1975-1990), tout comme le conflit en Irak ont fortement divisé les intellectuels arabes. L'image de l'Occident des «Lumières», porteur de la civilisation, a fait renaître dans les esprits de plusieurs intellectuels l'autre image, déjà présente depuis la fin du XIX $x^{e}$ siècle, celle d'un Occident colonisateur et impérialiste. Alors que pour d'autres, comme Kanan Makié (Al Hassan, 2005), les États-Unis constitueront le salut du monde musulman, qui pourrait sauver le monde arabomusulman du despotisme aveugle de ses dirigeants.

\section{Facteurs exogènes}

Ces facteurs sont dits exogènes car ils n'interviennent pas directement dans la logique sectaire, ni ne tiennent de place dans leurs discours.

\section{La crise du modèle étatique dans le monde arabo-musulman}

Ce facteur se rapporte à la nature de l'État post-colonial dans les pays arabes. Cette crise est tantôt militaire ou sécuritaire (policière), tantôt tribalo-clanique. Cela a mené à l'érosion de l'État de droit au profit de l'autoritarisme et du despotisme, et du coup à la marginalisation de la société civile ainsi qu'à l'accaparation des ressources, des richesses et du pouvoir. Par conséquent, cela a entravé, et entravera, le chemin vers l'instauration d'une démocratie véritable. La solution serait, à cet égard, non seulement l'établissement des mécanismes officiels de la démocratie (élections, référendums, etc.), mais aussi dans une reconsidération du rôle de l'État et de ses institutions dans laquelle une prééminence de la loi, la séparation des pouvoirs de l'État et de la religion, ainsi que le respect des libertés publiques et individuelles seraient assurés. Mais, dans l'état actuel des choses, et pour des raisons historiques et culturelles, l'ordre politique, dans plusieurs pays arabes de la région, reste incapable d'établir de telles réformes dans l'espace arabo-musulman. 
En effet, dans sa relation avec la société civile, l'État connaît un déficit de légitimité. Mais aussi, puisqu'il est oppresseur, l'État est incapable de monopoliser légitimement la violence; l'exemple de l'Égypte des années 1970 et 1980, où la violence a été pratiquée par plusieurs groupes islamistes radicaux, l'illustre bien. Dans certains pays, l'État est perçu comme un État-secte, ou un État-tribu. Dans ces cas, le monopole de la violence n'est pas tant celle de l'État, mais plutôt d'une secte; par exemple, en Syrie, les institutions étatiques sont considérées par la société civile - et le syrien moyen - comme étant usurpées par la communauté Alaouite du parti Baath ou encore par la famille Al Assad (elle-même Alaouite). En Irak, sous Saddam Hussein, le pouvoir était concentré entre les mains d'une minorité issue de Takrit, la ville natale de Saddam Hussein. Certes, il y avait aussi des Chiites et des Kurdes au sein de l'appareil étatique de cette époque, mais les postes clés étaient entre les mains des Takritis. Le Liban aussi a été perçu comme favorisant un groupe. Ainsi, pendant longtemps le Liban a été vu comme un État maronite: la maronité politique se voulant représentative des Chrétiens, en tant que corps social.

C'est ainsi que l'État, bien que se disant laïc, a lui-même été responsable de la formation de groupes sectaires, par réaction et opposition au groupe sectaire au pouvoir. Le cas de l'Irak est révélateur, où le Hizb ad-Da'wa chiite s'opposait au pouvoir répressif incarné par le Parti Baath. Au Liban, la représentation des communautés par des groupes confessionnels touche les quatre principales communautés, chiites, chrétiennes, druzes et sunnites, selon un phénomène de vases communicants. Cette mauvaise gestion de l'hétérogénéité politique et communautaire a beaucoup facilité l'instauration de la violence à caractère sectaire. Cela étant, quid maintenant de la situation dans les pays où la démocratie est embryonnaire, à l'instar de l'Irak actuel. Il n'est pas exagéré de dire, que dans ce cas, la démocratie est mise à rude épreuve. En effet, la promesse démocratique, puisqu'elle a été offerte dans un terrain qui n'y était pas prêt, a généré une abondance de demandes et de revendications sectaires que l'État, malgré sa nouvelle légitimité, n'a pu satisfaire. Par conséquent, les critiques ont mis en cause le gouvernement puisqu'il est considéré comme favorisant une communauté, principalement chiite, aux dépens des autres.

Par ailleurs, dans de telles démocraties émergentes où les périodes de violence vacillent entre continuité et rupture, de nouveaux critères de justice s'installent après la fin du règne de la terreur. Les canaux 
étatiques habituels de règlement des différends entre protagonistes sociaux sont évincés pour céder la place aux vendettas, qui alimentent la violence sectaire. Le cas de l'Irak actuellement en est un exemple frappant. En sus, quand cet État nouveau et fragile se trouve dans l'incapacité de distribuer équitablement les fonctions étatiques entre les groupes en présence, le monopole de la violence se trouve au centre des objectifs visés par ces groupes et communautés afin de sauvegarder leur supériorité et de demander d'autres privilèges et droits.

L'intervention étrangère: la persistance de la règle du "diviser pour régner» ou la persistance de la mentalité de conspiration

Dans un article intitulé «The redirection» (Hersh, 5 mars 2007), Seymour Hersh avance, sans être le premier ni le seul, que la nouvelle stratégie de la Maison Blanche au Moyen-Orient pousse toute la région dans un conflit sectaire élargi entre les musulmans chiites et sunnites. Cette idée est favorablement accueillie et fortement propagée et partagée au Moyen-Orient par plusieurs milieux diplomatiques, culturels et journalistiques de toutes tendances confondues, à droite comme à gauche, laïques ou religieuses. Particulièrement, le lien de causalité entre le déploiement militaire de l'armée étatsunienne et la survenue de la violence sectaire en Irak est ainsi expliqué. Entre autres, plusieurs quotidiens saoudiens ${ }^{4}$, généralement considérés comme "proétatsuniens», ne manquent pas de le souligner avec insistance.

Pour ces milieux, il ne fait pas de doute que la violence sectaire en Irak a commencé avec l'intervention étrangère, qui correspond aussi à l'effondrement de l'État central. Mais il serait trop facile d'imputer la violence sectaire en Irak aux seules forces étrangères. D'ailleurs, ceux qui privilégient le rôle de l'intrusion étrangère pour expliquer la survenue de violence sectaire se demandent pourquoi une telle violence ne s'est-elle pas déclenchée dans d'autres pays qui connaissent également une diversité confessionnelle et une présence étrangère (comme les pays du Golfe où il y a une présence militaire étatsunienne importante). Par contre, l'intervention étrangère pourrait, éventuellement, tirer profit de la violence sectaire en Irak. Des observateurs ${ }^{5}$ attribuent, en effet, à

4. Voir entre autres les éditoriaux des quotidiens saoudiens: Jazirah, ar-ryiad, 'okaz, al-wattan, du 11 avril 2007.

5. Voir entre autres Rubaiee (Al), Fadhil (2007) et Haseeb, Khair Ed-Din (2006). 
l'intervention étrangère des projets de balkanisation, de partage de l'entité irakienne ${ }^{6}$ ou encore de submersion de la résistance antiétatsunienne dans une violence de guerre civile.

\section{La quête du leadership régional}

Cette violence sectaire a d'autres raisons d'être, dont la quête du leadership au sein du monde musulman sur le plan religieux et politique, notamment par l'Iran chiite et l'Arabie saoudite wahhabite, constitue un élément certain. Sur ce point, on peut dire que la rivalité iranosaoudienne est au cœur même du remodelage du Grand Moyen-Orient. La stratégie étatsunienne y a alterné, à des tournants précis et délicats des convergences, avec chacune des puissances. Une fois pour sanctionner le rôle saoudien dans les événements du 11 septembre et plusieurs autres fois pour resserrer l'endiguement de l'Iran. La rivalité entre l'Iran et l'Arabie saoudite connaîtrait d'ailleurs des frappes sous la ceinture, par exemple, des Iraniens impliqués dans des attentats sur le sol saoudien et des pro-saoudiens soupçonnés de fomenter des troubles sur le sol pluriethnique iranien dans les communautés arabophones et sunnites.

Toutefois, Il serait inopportun de penser la rivalité irano-saoudienne au Moyen-Orient, en omettant plusieurs perspectives, dont l'idéologie, la culture, la géopolitique (à la recherche du leadership régional) et même l'économie. Certes, l'Iran et l'Arabie saoudite polarisent le monde musulman en deux faisceaux doctrinaux, sunnite et chiite, mais la rivalité irano-saoudienne dépasse l'antagonisme religieux et surtout confessionnel, sunnite et chiite, pour s'inscrire profondément dans le conflit régional depuis la révolution islamique en Iran en 1979, en vue d'une domination de la région du Golfe, une concurrence pour porter le flambeau de l'Islam ou en être son seul porte-parole crédible.

C'est, en effet, la thèse de la guerre par procuration entre ces deux pays qui y gagne en résonance. Le spectre de cette guerre «par procuration» en Irak soulève des craintes à Washington et à Bagdad. Surtout

6. La partition du pays dans un souci de fédéralisme va aboutir à un découpage ethnique permettant de constituer trois entités principales: Kurdo-sunnite au Nord, Arabosunnite au centre, et Arabo-chiite au Sud, ouvrant la possibilité aux autres minorités, même si elles ne sont pas représentatives, de réclamer des entités pures et des territoires propres. En sus, si ce découpage est favorable aux Kurdes, vu la richesse pétrolière du sol au Nord irakien, et les chiites au Sud, vu la richesse aussi du sol ainsi que la proximité avec l'Iran chiite, les sunnites se sentent largement lésés, étant donné que la nature a défavorisé le centre irakien (relativement au Nord et au Sud). 
à la suite des informations écoulées dans des déclarations officielles, parfois officieuses même, selon lesquelles l'Arabie saoudite serait prête à aider financièrement les arabo-sunnites irakiens, dans le cas d'un éventuel conflit ave les chiites, après le départ des Étatsuniens ${ }^{7}$. En effet, ce sont principalement l'Arabie saoudite, mais aussi la Jordanie et l'Égypte, qui font l'objet de ces craintes qui sont, par ailleurs, justifiées par l'influence iranienne croissante en Irak. Ces craintes sont, de surcroît, suscitées par la formation éventuelle d'un croissant chiite ${ }^{8}$, favorisée par l'entremise de l'Iran afin d'encercler les régions sunnites pour les affaiblir, mais aussi et surtout, pour satisfaire les ambitions nucléaires de Téhéran. Ces éléments soutiennent en effet la crainte que la majorité sunnite et la minorité chiite soient en quête du leadership régional au Moyen-Orient, ce qui constitue un facteur qui pourrait éventuellement alimenter la violence sectaire en Irak.

\section{Regards introspectifs sur la violence sectaire : l'éclairage des facteurs endogènes}

Il a été question plus haut des facteurs directs successibles de déclencher la violence sectaire dans la région du monde musulman. Mais la violence sectaire est un phénomène trop complexe pour n'être expliqué que par ces facteurs qui n'ont somme toute qu'un rôle de catalyseurs. D'autres facteurs fondamentaux, de nature structurale, sous-tendent également la violence. Les trois facteurs suivants en constituent les principaux. Ils concernent le patrimoine, une vision dualiste et exclusiviste du monde et enfin une idéologie de martyr.

\section{Le patrimoine mis en cause!}

La violence sectaire est perçue, à juste raison, comme la manifestation explosive d'une crise profonde de la culture arabo-musulmane, ce qui

7. Voir l'article de Nawaf Obaid paru dans le Washington Post à ce sujet (Obaid, 29 novembre 2006: A-23). Nawaf Obaid est conseiller au gouvernement saoudien et directeur de gestion du projet saoudien d'évaluation de sécurité nationale à Riyadh et adjoint au Center for Strategic and International Studies à Washington.

8. La géopolitique iranienne a suscité des craintes arabo-sunnites, surtout sur le plan des régimes en place que le roi hachémite jordanien Abdallah II a appelé, sans rectitude politique, le «croissant chiite» menaçant! Il signifiait cette ceinture de zone d'influence iranienne qui étrangle le monde arabe sunnite du côté de la région du Golfe jusqu'au Liban. Les mêmes craintes sont pressenties par les dirigeants en Arabie saoudite. 
constitue un premier facteur endogène contribuant à l'explication de la violence. En effet, le legs culturel arabe est basé principalement sur un fond tribalo-clanique dans lequel les liens sanguins priment sur l'individu; cela explique d'ailleurs le penchant pour le sacrifice de l'individu en faveur du groupe. En d'autres termes, en Islam, héritant des structures tribalo-claniques d'Arabie, l'individu se dilue dans l'esprit de la tribu, à l'opposé de la société libérale dans laquelle l'individu prévaut. Certes, l'Islam a connu un mouvement pour humaniser ce legs tribal (voir, entre autres, Boisard, 1980), mais la société arabo-musulmane, dans plusieurs de ses dimensions, est restée sous l'emprise de la mentalité tribalo-clanique dans laquelle la solidarité tribale, la vendetta et le despotisme dominent. La violence a certainement été inhérente à la vie humaine dans les sociétés archaïques. Elle a été un moyen d'exercer la domination ou encore l'acquisition des avantages politiques. D'une façon ou d'une autre, la violence a été utilisée comme moyen politique dans les guerres entre les nations, de même que comme moyen de résistance contre l'occupant, et lors de révolutions sociales et politiques, la violence a été utilisée comme moyen de lutter contre le despote. L'aspect belliqueux de la mentalité tribalo-clanique a constitué, en ce sens, un soubassement à la culture propagée par l'Islam. Ce caractère belliqueux se retrouve d'ailleurs dans le Coran à travers les versets du Jihad et la Sira, la vie du prophète, surtout dans ses guerres contre ses ennemis, comme le soutient l'historien Jean Flori (2002), qui avance que l'Islam en devient une religion de guerre ab initio. Flori ajoute que la notion de la guerre en Islam comme «conception conquérante et totalitaire» intègre même une vision dualiste du monde: un domaine de l'islam (Dâr al-Islam) et un autre de la guerre (Dâr al-Harb) (Ibid.: 108). C'est l'idée même qui sert encore de base aux idéologies qui promeuvent la violence sectaire dans la région arabo-musulmane.

Il faut ajouter que d'autres formes de violence sont aussi présentes dans les enseignements de l'Islam, soit dans les châtiments corporels ( $\left.h u d \hat{u} d^{9}\right)$,

9. Les hudûd sont des châtiments corporels ou des peines fixes visant précisément à empêcher les musulmans de commettre certaines irrégularités légales du point de vue de la Shari'a. Il y a dix hudûd correspondant à dix péchés principaux, quatre d'entre eux sont cités dans le Coran, tandis que les six autres sont tirés de la Sunna. Entre autres, les péchés de fornication (zina), d'ébriété ou de diffamation, sont passibles de lapidation (rajm), celui du vol qui requiert l'amputation de la main, tandis que les péchés d'apostasie (ridda), d'homicide, d'adultère ou d'homosexualité entraînent la peine de mort. Historiquement en islam, les budûd ont toujours suscité la controverse et leur application à la lettre n'était guère considérée. En 2005, l'islamologue Tariq Ramadan, dans un célèbre moratoire, a 
dans la violence contre les femmes ${ }^{10}$, dans la violence à l'égard des mécréants et des apostats ${ }^{11}$, dans le droit de les exécuter ou de les assassiner même par une initiative privée et enfin dans la violence contre soi-même, comme dans l'eschatologie chiite ${ }^{12}$. En ce sens, les premiers cas de violence sectaire dans l'histoire de l'Islam sont survenus lors de la Grande Discorde (Fitna) ${ }^{13}$, soit une période des troubles à la suite de l'assassinat du calife Othman, en 656. Les premiers qui l'ont pratiquée au nom de leur groupe sont les Kharijites ${ }^{14}$. Soutenus par une

appelé à la suspension des châtiments corporels pour être plus «fidèle au message de l'islam à l'époque contemporaine» (Ramadan, 2005).

10. On se réfère toujours à ce verset pour expliquer le droit d'un homme de frapper sa femme dans l'islam: «Les hommes ont autorité sur les femmes, en raison des faveurs que Dieu accorde à ceux-là sur celles-ci, et aussi à cause des dépenses qu'ils font de leurs biens. Les femmes vertueuses sont obéissantes (à leurs maris), et protègent ce qui doit être protégé, pendant l'absence de leurs époux, avec la protection de Dieu. Et quant à celles dont vous craignez la désobéissance, exhortez-les, éloignez-vous d'elles dans leurs lits et frappez-les. Si elles arrivent à vous obéir, alors ne cherchez plus de voie contre elles, car Dieu est certes, Haut et Grand! (Coran, 4:34). Mais ce verset est lui-même atténué par un autre: «Et parmi Ses signes Il a créé de vous, pour vous, des épouses pour que vous viviez en tranquillité avec elles et Il a mis entre vous de l'affection et de la bonté. Il y a en cela des preuves pour des gens qui réfléchissent.» (Coran, 30:21) D'ailleurs la tradition musulmane retient que le prophète Mahomet n'a jamais frappé une de ses femmes. Sans oublier l'interdiction par l'islam d'enterrer vivants des nouveau-nés du sexe féminin, une tradition bédouine préislamique d'Arabie.

11. La question de l'apostasie s'ouvre sur la notion d'Al walaa wa al baraa [L'alliance et le reniement]. L'alliance avec les autres fidèles musulmans qui inspire l'amitié et consolide l'entraide au sein de la communauté. Le reniement des apostats qui provoque l'inimitié, le rejet et la violence contre les infidèles (associationnistes ou ceux qui ont renié l'islam).

12. Les chiites commémorent avec des sentiments de culpabilité et de souffrance un événement marqueur de leur mémoire collective et de leur histoire tragique, celui de la décapitation de Hussein, fils d'Ali et petit-fils du Prophète, le 10 du mois du Moharram en 680 de l'année grégorienne, par les armées de Yazid Ibn Mouawia, second calife omeyyade. C'est le souvenir d'Achoura (dixième jour du premier mois du calendrier musulman, Moubarram). D'où les scènes violentes d'autoflagellation publiques annuelles quoique critiquées par des instances chiites.

13. Crise politique violente aux implications religieuses sous le califat d'Ali (656-661), et qui a brisé définitivement l'unité de la communauté islamique. Les sectes ou les courants en islam sont apparus pendant cette période cruciale.

14. Les Kharijites constituent avec les Sunnites et les Chiites l'une des trois principales branches de l'Islam. Cette branche a refusé l'arbitrage entre Ali et Mouawia à l'issue de la bataille «Siffin» qui les avait opposés en 657. Ali y a accepté l'arbitrage pour arrêter la guerre. Au début les Kharijites soutenaient Ali, mais après l'acceptation de l'arbitrage, ils se sont retirés et ont condamné les deux camps (celui de Mu'âwîya et celui d'Ali). Leur slogan est resté célèbre: "la bakimia illa lillab» (l'arbitrage n'appartient qu'à Dieu). Ses membres ont eu recours à la violence politique. L'un d'entre eux, Abdul Rahman Ibn Moljem, a été l'assassin du calife Ali, qui a lui-même massacré les Kharijites dans la bataille de Nahrawan en 658. Le Kharidjisme est surtout connu par son rigorisme poussé à l'extrême. Il s'est divisé, lui aussi, en plusieurs courants, le plus célèbre est celui des Ibâdites qui est présent à Oman jusqu'à nos jours. 
interprétation rigoriste du texte coranique, ils ont autorisé la violence contre l'autre, le non kharijite. Au xi siècle, une autre secte, les ismaéliens, dits les Assassins, sous la direction de Hasan-al-Sabbab (Le vieux de la Montage) pratiqua le terrorisme sectaire afin de renverser l'empire sunnite. La secte des Assassins terrorisa pendant longtemps l'empire sunnite dominant ses princes et ses califes «proches ou éloignés, ainsi que les seigneurs chrétiens voisins» (Lewis, 1984: 37). Une violence sectaire contre un pouvoir sectaire! L'histoire ne veut plus manquer des exemples. Ce serait gravé dans les imaginaires collectifs et individuels. De tels exemples sont d'autant plus pertinents que plusieurs slogans proclamés par les groupes sectaires et extrémistes religieux actuels relèvent de la même idéologie. Ces groupes, à l'instar de leurs ancêtres, puisent la justification de leurs actions dans le Coran, le Hadith et la biographie du prophète. Il n'est d'ailleurs pas étonnant de voir le même verset utilisé par deux groupes ennemis afin de justifier la violence contre l'autre.

\section{Une vision dualiste du monde et exclusiviste de l'Autre}

Le deuxième élément endogène expliquant en partie la violence sectaire dans le monde arabo-musulman est la vision dualiste qui consiste à considérer le monde divisé entre le mal et le bien, le vrai et le faux, le Halal et le Haram, soit le licite et l'illicite. Cette vision dans laquelle chaque protagoniste se définit comme détenteur de la Vérité Absolue mène naturellement à l'extrémisme et à l'intégrisme et est élaborée au niveau de la pensée pour passer en pratique dès que les circonstances le permettent ${ }^{15}$. Cette pensée dogmatique consiste en un rejet catégorique de la différence et du pluralisme; dans ce sens, la terminologie utilisée dans le discours puise dans des mots provocateurs tels que l'Autre impie, le traître, etc. Ainsi, dans la langue métaphorique, le monde qui se situe en dehors de la vision partagée par le groupe est vu comme diabolique, ou est dit vivre dans la jabiliya (l'ignorance et les ténèbres) alors qu'au sein du groupe, de l'intérieur, il est dit que la lumière règne sur les adeptes. Dans cette vision des choses, celui qui adhère à ce type de pensée, vécue comme une vérité, croit aussi toujours avoir raison. Toute autre vision du monde est déclarée fausse, la différence est jugée inacceptable, car elle est vue comme erronée. Le dialogue

15. C'est justement à ce moment qu'interviennent les facteurs qu'on a appelés exogènes. 
est donc vain; on dira qu'il est haram, soit illicite chez les extrémistes d'Al Qaïda en Irak, par exemple. La violence devient ainsi la seule solution envisagée pour mater toute divergence et imposer ses croyances dogmatiquement.

Le contexte présenté est semblable à celui «d'un retour au religieux qui se caractérise souvent par des lectures psychologisantes voire des lectures fantaisistes des grands textes religieux de l'humanité», avec une fétichisation des textes sacrés et une sorte de bricolage idéologique et identitaire (Römer, 1998: 12). Les discours sectaires deviennent salutaires puisqu'ils sont porteurs d'un message de délivrance. Leur rhétorique ne peut donc qu'exacerber la rupture du lien entre le citoyen et l'État-nation. C'est ainsi que la violence de la logique sectaire exclusiviste est conçue comme appartenant à la Communauté promise au Salut (Al-Firqa al-Najiya). Cette idée d'Al-Firqa al-Najiya suffit d'ailleurs à elle seule à justifier l'éradication de l'Autre. C'est ainsi que la violence sectaire répond à l'angoisse identitaire, qui constitue un des aspects de la défaite musulmane devant la civilisation occidentale, selon Arnold Toynbee qui ajoute qu'une telle angoisse est accentuée par l'héritage colonialiste ${ }^{16}$.

Dans cette perspective des dynamiques communautaires et confessionnelles, l'identité n'est plus patriotique ou même musulmane, même si elle s'approprie l'islam et parle en son nom, elle est plutôt confessionnelle et communautariste. Ce qui signe sa différence d'une rhétorique à forte connotation ethno-religieuse. Dans le cas de l'Irak, la résistance contre les Étatsuniens est plutôt vue comme une réalité chiite ou encore sunnite. Au Liban, on parle du Hezbollah comme d'une résistance chiite. La connotation est donc confessionnelle, et son discours cohésif reste une tentative de justifier la violence au nom du groupe. Lappartenance à un groupe est parfois recherchée aussi pour des fins d'autosuffisance financière, car il devient une source de financement pour les adhérents, comme cela a été le cas pendant la guerre du Liban. D'ailleurs, le droit de quitter le groupe, comme un parti unique avec un chef sanctifié, devient impossible. Ainsi, les loyautés sectaires ne doivent pas être vues comme des épiphénomènes ou des accidents, mais plutôt comme des faits sociaux entiers. 
Une tendance autodestructrice enveloppée d'une idéologie du martyre

Le troisième facteur endogène participant à la violence sectaire consiste en une cacophonie des appels à la violence, justifiés religieusement, et qui constitue un des symptômes troublants, l'on pourrait même dire répulsifs, de l'islam politique. L'absence d'autorité religieuse unique et centrale, par exemple, est largement responsable de cette anarchie politique. D'où cette audace surprenante à recourir à la violence au nom de Dieu. La martyrologie, ou al-Istichbad, devient en effet une idéologie et un moyen privilégiés de militantisme islamiste. Au cours des dernières décennies, cette martyrologie domine les écrits des islamistes activistes, mêmes chez les sunnites. Certes, le genre d'opérations-suicides qu'elle commande, en dépit du fait que le suicide soit prohibé dans l'islam est un fait historique peu commun dans l'histoire musulmane, dont l'ampleur et l'absence de discernement dans le choix des victimes (civils, femmes, enfants) et dans les cibles (universités, mosquées, hôpitaux) en font des actions actuelles dont on ne peut nier l'évidence. Néanmoins, la martyrologie, mise en évidence plutôt dans la tradition chiite, n'a pas traditionnellement été centrale dans la vision politique islamique dominante. Par contre, il est certain que le texte religieux, qu'il soit le Coran, ou la Sunna soit la «règle de conduite» du Prophète, ses paroles et ses actes, reste le référent principal de cette idéologie de l'istichhad (martyre ou martyrologie).

Un badith ${ }^{17}$ du prophète attribuerait la faiblesse des musulmans ou leur impuissance à "la vénération de la vie et la haine de la mort» (bobou al-bayat wa karabyiatou al-maout). Ce badith est largement utilisé dans la littérature activiste islamiste, d'abord pour constater la dégénérescence ou l'état de faiblesse de l'Umma. Puis, il est utilisé pour sortir de ce qui est vu comme un état de torpeur de l'islam, en l'inversant: mépris pour la vie et vénération de la mort. D’où une fétichisation des textes religieux par les islamistes radicaux, avec une lecture littérale et métaphorisante qui aboutit à une interprétation autre du suicide, qui le permet, bien qu'il soit catégoriquement interdit dans l'islam.

L'istichhad (martyrologie) spectaculaire des voitures et des ceintures piégées s'est répandue dans la région, tactique importée d'ailleurs,

17. Terme qui désigne les paroles et les actes du prophète Mahomet rapportés par ses compagnons, dans le but de constituer le modèle à suivre par tous les musulmans. L'ensemble des hadiths reconnus comme authentiques constitue la seconde source de la Shari'a (ou Chari'a), après le Coran. 
comme un moyen de résistance à l'occupation israélienne. Il s'est mis en route, activé par la thèse «nous aimons la mort plus que les juifs n'aiment la vie ${ }^{18}$ ». Dans le cas de la violence sectaire, l'idée que «nous aimons la mort plus que l'Autre n'aime la vie» est ainsi advenue. L'Autre étant le mécréant qui ne fait pas partie du groupe, qu'il soit sunnite, chiite, non pratiquant ou autre. La violence sectaire prend alors la forme du jihad, notion devenue tellement ambiguë qu'elle nécessite des éclaircissements. Étymologiquement, le mot arabe jibad veut dire «effort accompli en vue de la réalisation d'un objectif»; toutefois, il faut faire une distinction entre le «grand jibad» et le «petit jibad». Le premier, le grand jibad, doit être mené par le musulman en vue d'une purification spirituelle, en s'appliquant scrupuleusement dans les actions du prophète. Le deuxième, le petit jibad, est le combat «saint» et guerrier que les musulmans doivent mener pour se défendre (s'il est défensif) de leurs ennemis et s'imposer (s'il est offensif) à eux. Depuis les années 1980, la primauté est donnée à cette deuxième forme du jihad, qui apparaît comme la condition nécessaire pour sortir de l'emprise de la tutelle étrangère, le cas de l'Afghanistan pendant les années 1980 en est l'exemple type. Cette seconde forme du jibad qui est certes la plus retenue par l'inconscient collectif occidental a naturellement évolué à travers le temps et relativement aux circonstances historiques ${ }^{19}$.

De l'obligation rituelle que lui a conférée la tradition au début des guerres entre la petite communauté musulmane entourant le prophète et celle de l'aristocratie Qoraichite, alors non convertie à l'islam, jusqu'à son érection comme fondement de la religion musulmane, la notion du jibad a été largement extrapolée, voire instrumentalisée par l'activisme jihadiste. Dans le cas de la violence sectaire, le jibad sert en effet à justifier les opérations suicides commises au nom du groupe, mais qui sont vues dans l'idéologie sectaire comme étant perpétrées au nom de Dieu et de sa religion. Cela étant, bien qu'elle ait été d'abord utilisée contre «l'ennemi», cette violence a par la suite été tournée contre soi, contre un citoyen ou un coreligionnaire d'une autre confession. À cet effet, les symboles religieux sont les principales cibles des terroristes de part et d'autre des confessions. À titre d'illustration, ceux des sunnites

18. Voir à titre d'exemple l'article de Charles Moore (18 décembre 2004) paraphrasant Azzam Tamimi, directeur de l'Institut de la pensée politique islamique de Londres.

19. Pour plus de détails, voir le chapitre «La violence politique au nom de l'islam» dans Aoun, 2007: 119-140. 
ont été les mosquées Anas Ibn Malik et d'al-mo'alemayne brûlées à Bagdad, alors que ceux des chiites ont été les mausolées des Imams chiites à Karbala et Nadjaf entre autres.

Toutefois, un autre point doit être mis en exergue; c'est que ce moyen de destruction qui constitue certes aussi un moyen d'autodestruction n'aurait pas trouvé écho au sein de la jeunesse des pays arabo-musulmans si les circonstances ne l'avaient pas favorisé. En effet, plusieurs auteurs et analyste ${ }^{20}$ estiment que c'est principalement la pauvreté, le chômage, le pessimisme et l'impuissance à changer sa situation qui constituent un terrain fertile pour l'instauration de l'idéologie de l'istichbad au sein d'une jeunesse qui dénonce par de tels actes sa vie miséreuse.

D'autres penseurs (voir par exemple Al Ansary, 15 avril 2004) estiment, à l'opposé, que de tels agissements sont dus à une culture de la haine et à la présence d'une pensée rancunière, hostile à toute différence et malveillante telle qu'elle se manifeste dans certains écrits. Ces penseurs sont, en effet, d'avis que ni le despotisme, ni l'autoritarisme, ni la situation économique ${ }^{21}$ puisque c'est seulement dernièrement qu'elle a été mise en exergue dans leurs discours, ni l'alignement des États-Unis au côté d'Israël puisque la question palestinienne n'est pas dans les priorités des groupes en Irak, ni l'ignorance - arguant que des Ulémas qui prônent et appuient de tels actes de martyres ne peuvent être ignares - ni non plus le manque de liberté dans les sociétés arabo-musulmanes puisque les gens qui adhèrent à une telle pensée destructive ne se préoccupent guère, voire point de la liberté des autres, ne peuvent être considérées comme des causes de la violence sectaire actuelle.

D'autres penseurs ${ }^{22}$ encore estiment que la violence sectaire prônée par les mouvements radicaux puise ses racines dans des interprétations abusives ou erronées des versets coraniques, sans égard à leur contexte

20. Voir l'intégralité du débat avec Damien Millet, président du Comité pour l'annulation de la dette des pays du tiers-monde (CADTM), intitulé «Pays pauvres: le G8 en fait-il assez? » et organisé le 8 juillet 2005 par le quotidien français Le Monde. Disponible sur <http://www.lemonde.fr/web/chat/0,46-0@2-3220,55-669307,0.html>

21. À cet effet, si on peut dire que la pauvreté ne constitue pas un facteur dans le cas du Maroc, après les bombardements à Casablanca le 16 mai 2003, la situation est tout autre en Arabie saoudite, où le problème de la pauvreté ne se pose pas de la même façon (cela ne signifie pas qu'il n'y a pas de pauvreté en Arabie saoudite) qu'ailleurs dans l'espace musulman. L'Arabie saoudite est un pays riche, mais des Saoudiens ont rejoint les rangs d'Al Qaïda en Irak pour combattre les Étatsuniens et les Chiites.

22. Voir entre autres le livre de Youssef Al Qaradaoui Fatwas contemporaine (Qaradaoui, 1993). 
historique ${ }^{23}$. Par ailleurs, on pointe du doigt surtout les Ulémas contemporains à cause de leurs positions ambiguës sur la question, de leur silence suspect ou de leur mutisme "complice», du moins dans le sens qu'ils ne mettent pas en exergue le danger des interprétations personnelles des versets du Coran invoquant cette notion du jibad et de violence (voir à titre d'exemple Jabri, 2003). Cette réticence silencieuse à prendre position des Ulémas contemporains, couplée aux mauvaises interprétations du texte coranique, ont contribué dans les esprits, surtout occidentaux, à ériger une image négative de l'islam. Mais, au-delà de l'image, cette absence d'implication est de nature à justifier la Fitna, à la propager et même à la rendre menaçante pour l'ordre étatique et social dans la plupart des sociétés musulmanes. Aussi, cela serait un obstacle majeur pour l'édification d'un État moderne.

\section{Discours et méthodes de la violence sectaire: le cas de l'Irak}

Les promoteurs de la violence sectaire entretiennent des discours cohésifs, fermés, articulés notamment par les fatwas exclusivistes ou les fatwas d'anathème (takfir). Le dénigrement de l'Autre reste l'idée de ralliement de ces discours. Cela est accentué d'une rhétorique de victimisation qui favorise une lecture de sa propre histoire selon laquelle tous les musulmans sont attaqués injustement et de celle d'une théorie du complot par laquelle on n'est jamais responsable de son propre sort, ainsi que d'une vision apocalyptique du monde, c'est-à-dire celle d'un combat inévitable entre le Bien et le Mal. Mais, ce qui retient l'attention dans ces discours, c'est le retour en force de la rhétorique du takfir. Ce n'est pas une innovation, elle prend plutôt ses sources aux premiers temps de l'islam, surtout au temps de la grande discorde: la Fitna. La méfiance historique et réciproque entre sunnites et chiites, depuis les événements sanglants de la Fitna, en constitue une des figures déterminantes. Cependant, la situation n'a guère changé depuis ce temps. Le takfir, ou fatwa d'anatbème, vise généralement tous les non-musulmans, mais aussi d'autres courants de l'islam ainsi que des personnalités publiques et des musulmans non pratiquants qui sont jugés comme des faux musulmans.

23. Dans cette perceptive, Youssef Al Qaradaoui (référence sunnite) et Mohamed Hussein Fadlallah (référence chiite) ont émis des fatwas pour interdire aux sunnites et aux chiites de s'entretuer. 


\section{Le discours exclusiviste d'Al Qaïda dans la société multiethnique irakienne}

La question de l'anathème ouvre sur une autre plus controversée: l'autorisation fondée religieusement de tuer l'apostat et de spolier ses biens. Selon la théorie dite de l'istiblal, utilisée notamment et couramment par Al Qaïda en Irak, le combattant islamiste pourrait se munir de ce privilège religieux qui consiste à considérer l'action de tuer l'ennemi et de spolier ses biens comme permise et licite dans l'islam ou halal (d'où le substantif istiblal). Cette théorie est utilisée par certains groupes jihadistes. Bien qu'il s'agisse d'une déformation des préceptes de la Sharia, elle est utilisée pour justifier la perpétration du jibad à travers des actes de terrorisme et de massacre de femmes, d'enfants et des non-combattants, qu'ils soient ou non musulmans. L'istiblal légitime toutes les tactiques du combat contre l'autre et les rendent halal et licites, qu'il s'agisse de nettoyage confessionnel, d'enlèvement identitaire, de liquidation, de lynchage, de viols, d'attentats suicides, par exemple dans les lieux sacrés chiites. En outre, les chrétiens se voient aussi imposés la jizya et il arrive que leurs biens soient vus comme des butins (ghanima).

La théorie de l'istiblal rejoint une autre notion mise en évidence dans la littérature jihadiste, surtout celle d'Al Qaïda en Irak, c'est la notion de tatarrouss $^{24}$. Les activistes islamistes s'y réfèrent pour légitimer le massacre de civils en Irak. En effet, Al Qaïda en Irak autorise l'attaque de ses ennemis non musulmans, même s'ils utilisent des civils musulmans comme boucliers humains. Encore faut-il ajouter que la même notion a été prônée par les Groupes Islamiques Armés (GIA) en Algérie durant les années 1990. Rappelons aussi que plusieurs interprétations qui vont dans ce sens se réclament de l'islam rigoriste d'Ibn Taymiya ${ }^{25}$ et se réfèrent à ses écrits.

24. La notion d'at-tatarouss signifie que pour le bien de la communauté musulmane et la protection de la religion (qui passe avant la protection de l'être humain), certains Ulémas ont autorisé d'attaquer l'ennemi s'il utilise des civils musulmans comme boucliers humains. Ces derniers, une fois morts, sont considérés comme des martyrs, tués pour la noble cause.

25. Ibn Taymiya [1263-1328] est un théologien musulman et un des plus grands jurisconsultes de l'islam, apparenté à l'école hanbalite rigoriste. Il est l'auteur de nombreux ouvrages religieux, dont Dar'u ta'ârudb il-aqli wan-naql [Réfutation de l'opposition entre raison et révélation], et $A t$-Tawassulu wal-wacîlab [Intercession licite, intercession interdite] (notamment en ce qui concerne le culte des saints). Ibn Taymiya est appelé Cheikh al-Islâm, un titre parmi les plus hauts donnés aux Ulémas de l'islam. 
Brigades Badr et armée al Mahdi: la rhétorique de la haine des milices chiites

Ainsi, la crainte transformée en haine de l'Autre prime. Les deux rivaux, sunnites et chiites, engagés dans des cycles de violence sectaire produisent des discours idéologiques centrés sur la justification de la violence contre l'Autre. L'Autre est à la fois frère et ennemi, loyal et traître, bon croyant et faux musulman. Dans l'état actuel des choses, les attentats contre les quartiers et les mosquées sunnites, notamment Al A'zamyia à Bagdad, sont attribués ${ }^{26}$ principalement aux groupes chiites armés soit les brigades Badr et l'armée al Mahdi. La violence sectaire de ces deux groupes chiites est liée à la frustration ressentie généralement par la communauté chiite à travers l'histoire musulmane. En effet, l'imaginaire chiite retient avec force l'usurpation du pouvoir par les sunnites depuis les premiers califes, à partir de 632 , date de la mort du prophète Mahomet. De plus, leur persécution sous le régime batthiste dirigé par Saddam Hussein a suscité une grande colère. Les sunnites sont qualifiés de Nawasib, terme utilisé par les chiites pour désigner les sunnites qui leur sont hostiles. Ainsi, tous les facteurs sont réunis pour l'instauration d'une violence sectaire en Irak.

\section{Conclusion: réflexions sur une sortie de la spirale de la violence sectaire dans l'espace islamique}

Après ces développements, plusieurs questions s'imposent. D'abord, quelle gestion propose l'Islam, ou de quels mécanismes se dote-t-il pour mettre fin à la violence sectaire? Dans cette perspective, il faut noter que les penseurs de l'Islam ont toujours eu cette difficulté d'atténuer l'opposition entre deux tendances qui se tiraillent entre l'exagération, al-ghoulou, et la modération, al-wasatiya. L'islam simple pratiqué par la majorité des musulmans est généralement perçu comme prêchant al-wasatiya, mais ce sont les cas d'al-ghoulou en islam et leurs dérapages vers la violence qui ont largement été retenus par l'histoire de l'Islam.

26. Ces attentats sont dénoncés, entre autres, par Iraq Freedom Congress (Le Congrès des libertés en Irak), parti politique irakien qui regroupe plusieurs organisations, associatives et syndicats irakiens de différentes idéologies. L'IFC est un fervent défenseur de l'idée du départ des troupes étatsuniennes de l'Irak. Voir le communiqué sur le lien suivant: $<$ http://www.ifcongress.com/English/News/Feb06/shrine-bombs.htm> 
Puis, qui a le monopole de la violence en Islam? Le débat est autour de wali al $\mathrm{Amr}^{27}$, soit le responsable de la chose publique en terre d'Islam. Mais il faut préciser que dans la théorie politique musulmane, élaborée principalement par des théologiens et des conseillers des califes et des rois, soit Ghazali (1058-1111), Ibn Taymiya (1263-1328) et Al Mawardi (972-1058), le fait d'accepter un wali al Amr, même s'il est un despote ( $\left.j a^{\prime} i r\right)$, par peur du vacuum politique, justifie les violences commises par celui-ci au nom de l'islam. Ces questions étant posées, plusieurs constats sur le phénomène de la violence sectaire dans l'espace islamique peuvent être faits. Il est à remarquer d'abord que la citoyenneté, notion centrale de la modernité, ne s'est pas imposée comme lien qui prime sur les appartenances religieuses et confessionnelles. Les loyautés primaires, qu'elles soient religieuses, communautaires, groupales, tribales ou claniques, ont ainsi prévalu sur la loyauté à l'État. Celui-ci est d'ailleurs considéré comme artificiel et imposé par les volontés étrangères coloniales.

Mais aussi, la disparition ou le rétrécissement de l'écart entre l'État et la société civile, dont l'autonomie est limitée par l'oppression et le pouvoir autoritaire, doit être soulignée. D'où le fait que l'effondrement de l'État dictatorial, par exemple celui de l'Irak, lui-même fondé sur la violence sectaire, a abouti à la fin de l'État et à la fin de la politique connue sous la catégorie de l'État failli. Ainsi, à l'heure de la mondialisation et du néolibéralisme, l'État autoritaire s'accroche aux moyens sécuritaires pour combler ses failles et ses défaillances sur les plans économiques et sociaux. C'est dans ce contexte que la montée des associations religieuses et des groupes tribalo-claniques devient une réalité préoccupante pour l'État puisque ces groupes deviennent de fait des refuges et des instances d'autodéfense contre la violence étatique et constituent des solutions de rechange même à l'échec de l'État dans sa fonction de veiller au bien-être de ses citoyens. Les partis religieux, en Irak par exemple, ont été une réponse contre l'autoritarisme de Saddam Hussein et sa laïcité autoritariste. Ces partis religieux sont sollicités par de nombreux citoyens pour combler des besoins vitaux et pour assurer des mécanismes d'autodéfense identitaire.

27. Cette notion a beaucoup changé à travers le temps et aujourd'hui, wali al Amr peut être identifié au haut responsable politique : émir, sultan, roi ou président... Ainsi en Islam, le recours à la violence légitime est une décision de wali al Amr; sinon, une loi fondamentale de la Chari'a qui est l'obéissance à wali al Amr est transgressée. 
Enfin, on ne peut omettre de signaler le point fondamental pour sortir de ce labyrinthe sectaire: c'est le retour d'un État civil fonctionnel, soit un État de droit doté de mécanismes de résolution de conflits, et détenant le monopole exclusif de la violence dans la société et d'y recourir avec circonspection dans des cas isolés et dans le respect des lois. Pour y arriver, il faudrait idéalement éliminer le confessionnalisme en interdisant aux groupes à connotation religieuse et confessionnelle toute possibilité de représentation politique. Il ne s'agit pas d'interdire les confessions religieuses par le fait de la transition démocratique ou par l'imposition de la laïcité. La violence sectaire serait apaisée ou mise sous contrôle par l'application de la démocratie consociative gérée par un État de droit et fondée sur la priorité de la raison d'état. L'Islam a certes des mécanismes de rapprochement entre ses branches et écoles, mais il ne peut pas avancer de solutions hors des acquis de la modernité. Ces acquis, soit la citoyenneté, la liberté individuelle et l'égalité doivent de surcroît être assujettis à un État de droit mené par des élus qui acceptent le principe de l'alternance du pouvoir.

Il est bien manifeste que la violence sectaire est due à des situations de transition du fait de l'effondrement d'un régime politique autoritariste, d'un ordre sociopolitique particulier et de facteurs d'accentuation de la crise. Au cours de cette transition historique, les composantes religieuses et ethniques en lutte entre elles recourent à la violence pour confirmer leurs gains ou encore défendre leurs privilèges menacés. Il importe enfin que dans cette période de mondialisation, les élites musulmanes, dans leurs foyers d'origine ou dans l'espace culturel occidental, se sentent interpellées de plus en plus pour répondre aux exigences de l'interculturalité et surtout à celles de la critique de la violence sectaire et de son argumentaire fondé sur les textes sacrés.

\section{Références}

Al Hassan, B. (2005). Thaqafat al istislam (La culture de la capitulation). Beyrouth: Riad El-Rayyes Books.

Al Ansary, A. H. (15 avril 2004). Jihad ou Terrorisme?... Comment les néokharidjites ont défiguré la notion du jihad en islam. Al asharq al Awsat, 9270, p. 9.

Aoun, S. (2007). Aujourd'bui l'Islam: Fractures, intégrisme et modernité. Montréal: Éditions Médiaspaul.

Bennabi, M. (1990). Le problème des idées dans le monde musulman. Alger: El Bay'yinate. 
Boisard, M. (1980). L'bumanisme de l'islam. Paris: Albin Michel.

Boutaleb, A. (1995). Le monde islamique et le projet du nouvel ordre mondial. Paris: PUF.

Charara, W. (Juillet 2005). Effervescence au Proche-Orient: Instabilité constructive. Le Monde Diplomatique, p. 9.

Flori, J. (2002). Guerre sainte, jibad, croisade. Violence et religion dans le christianisme et l'Islam. Paris: Seuil.

Haseeb, K. E. (2006). Al-'iraq: mina al-ibtilal ila at-tabrir (L'Irak: de l'Occupation à la Libération). Beyrouth: Markaz Dirassat al Wahda al Arabiyya (Centre des études de l'unité arabe).

Hersh, S. M. (5 mars 2007). The Redirection: Is the Administration's new policy benefitting our enemies in the war on terrorism? The Newyorker. Disponible sur:

<http://www.newyorker.com/reporting/2007/03/05/070305fa_fact_ hersh>

Jabri, M. A. (2003). La violence et l'accommodement idéologique. Mis en ligne le 10 juin 2003 : < http://www.aljabriabed.net/terrorism3.htm>

Lewis, B. (1984). Les Assassins. Terrorisme et politique dans l'Islam médiéval. Bruxelles: Éditions Complexe.

Mayeur-Jaouen, C. (2002). Saints et héros du Moyen-Orient contemporain. Paris: Maisonneuve \& Larose.

Moore, Ch. (18 décembre 2004). It is Muslims who have most to fear from Islamists. The Daily Telegraph. Disponible sur:

<http://www.telegraph.co.uk/opinion/main.jhtml?xml=/opinion/2004/ 12/18/do1801.xml>

Obaid, N. (29 novembre 2006). Stepping Into Iraq: Saudi Arabia Will Protect Sunnis if the U.S. Leaves. Whashington Post, p. A-23.

Qaradaoui (Al), Y. (1993). Fatwas contemporaine (Tome I). Al Mansoura: Dar al wafaa li tibaâa wa an-nachr wa tawziâ. Disponible sur:

$<$ http://www.qaradawi.net/site/topics/article.asp?cu_no $=2 \&$ item_no $=1$ 96\&version $=1 \&$ template_id $=8 \&$ parent_id $=12 \#$ top $>$

Ramadan, T. (2005). Appel international à un moratoire sur les châtiments corporels, la lapidation et la peine de mort dans le monde musulman. Disponible sur:

<http://www.tariqramadan.com/spip.php?article258>

Rodinson, M. (1972). Marxisme et monde musulman. Paris: Seuil.

Römer, T. (1998). Dieu obscur. Le sexe, la cruauté et la violence dans l'Ancien Testament. Genève: Labor et Fides.

Rubaiee (Al), F. (2007). Ma ba'd al-istichrak: al-ghazou al-amriki lil-'iraq wa 'aoudat al-colonialiat al-bayda'a (Post-Orientalisme: Invasion américaine de l'Irak et le retour des Colonialismes blancs). Beyrouth: Markaz Dirassat al Wahda al Arabiyya (Centre des études de l'unité arabe).

Toynbee, A. J. (1948). Civilisation on Trial. Londres: Oxford University Press.

Tueni, G. (1985). Une guerre pour les autres. Paris : Éditions JC Lattès. 
ABSTRACT - The violence in some corners of the Muslim world reveals serious flaws in the social contract. The outbreak of sectarian violence brings into question the soundness of the process of modernizing state. The Arab-Muslim culture admits symptoms of factors that make it incubator of sectarian violence; this later is considered as a crisis of the Arab-Muslim culture, since its formation. A dualistic vision of the world and exclusivist of the Other, in which fundamentalists resort to exclusivist arguments and demonize others. In addition, an ideology of martyrdom, where the praise of life has been overshadowed by the praise of death, and failure, or even model state collapse in this region, and also foreign intervention, which may partly explain the establishment of such violence.

Several findings on sectarian violence phenomenon in the Islamic space: The citizenship, central notion of modernity, has not imposed as a link, which takes precedence over the religious and confessional allegiances. The primary loyalties (religious or tribal) have prevailed over loyalty to the state. Disappearance or narrowing of the gap between the state and civil society. It is within this context that the rise of religious associations and tribal groups becomes a reality concern for the state. These groups become refuges and instances of self against state violence and are even alternatives to the failure of the State in its function of ensuring the well-being of its citizens. The fundamental point to get out of this "sectarian labyrinth" is the return of a state of law with conflict resolution mechanisms, and holding the exclusive monopoly of violence.

RESUMEN - Los actos de violencia en algunos rincones del mundo musulmán revelan graves fallas en el contrato social. La eclosión de la violencia sectaria pone en duda la solidez del proceso de modernización del Estado. La cultura árabe-musulmana padece sintomas que propician la incubación de la violencia sectaria: esta última es percibida como una crisis de la cultura árabe-musulmana desde sus inicios. Entre estos sintomas figura una visión dualista del mundo y excluyente del Otro en la cual los promotores de la violencia sectaria recurren a argumentos excluyentes y a la satanización del Otro. $A$ esto se añade una tendencia autodestructiva envuelta en una ideología del martirio en que el elogio de la vida es eclipsado por el elogio de la muerte. Sin olvidar, por otra parte, la constatación del fracaso del modelo de Estado en la región. Agreguemos a eso la intervención extranjera, especialmente la estadounidense en el caso de Irak, que puede explicar, parcialmente, la instauración de dicha violencia.

El artículo señala algunas otras constataciones sobre el fenómeno de la violencia sectaria. La ciudadanía, noción central de la modernidad, no ha conseguido convertirse en un vínculo que prime sobre las pertenencias religiosas y confesionales. Así, las lealtades primarias (religiosas, comunitarias, tribales o de clan) han prevalecido sobre la lealtad al Estado y esto se evidencia en la desaparición o la reducción de la brecha entre Estado y sociedad civil. En ese contexto, el auge de las asociaciones religiosas y de grupos clánico-tribales se ha vuelto una realidad preocupante para el Estado. Estos grupos se convierten en refugios o instancias de autodefensa contra la violencia del Estado y constituyen, incluso, alternativas al fracaso del Estado en su función de velar por el bienestar de sus ciudadanos. El punto fundamental para salir de ese laberinto sectario es la vuelta a un Estado civil funcional, es decir, un Estado de derecho dotado de mecanismos de resolución de conflictos y que detente el monopolio exclusivo de la violencia. 